\title{
The epidemiology of Taenia spp. infection and Taenia solium cysticerci exposure in humans in the Central Highlands of Vietnam
}

\author{
Dinh Ng-Nguyen ${ }^{1,2^{*}}$ D, Mark Anthony Stevenson ${ }^{1}$, Kathleen Breen ${ }^{3}$, Trong Van Phan ${ }^{4}$, Van-Anh Thi Nguyen ${ }^{2}$,
}

Tinh Van Vo ${ }^{5}$ and Rebecca Justine Traub ${ }^{1}$

\begin{abstract}
Background: Vietnam is endemic for taeniasis and T. solium cysticercosis. Despite this, information on the epidemiological characteristics of the diseases in the Central Highlands of Vietnam are poorly described. The aims of this study were to determine the epidemiological characteristics of taeniasis (Taenia spp.) and T. solium cysticerci exposure in humans in Dak Lak province in the Central Highlands, Vietnam.

Methods: This cross-sectional study was carried out in six villages in three districts of Dak Lak. A total of 190 households were visited. From each household, between one and five individuals were asked to donate a single faecal and blood sample and respond to a questionnaire. Serum samples were subjected to lentil lectin purified glycoprotein enzyme-linked immunoelectrotransfer blot assay to detect antibodies against T. solium cysticerci. Multiplex real-time PCR was used to detect Taenia spp. infection in faecal samples. A fixed-effects logistic regression model was developed to identify factors associated with the probability of Taenia spp. infection or T. solium cysticerci exposure risk. The contribution of each of identified factor was quantified using population attributable fractions.

Results: The prevalence of seroexposure to T. solium in Dak Lak was 5\% (95\% Cl 3\% to 8\%). Consumption of raw vegetables, sourcing drinking water from lakes, streams or ponds and the practice of outdoor defaecation were identified as primary risk factors for the prevalence of $T$. solium cysticerci exposure, while consuming undercooked pork and beef, pork tongue and observing Taenia proglottids in stool were associated with Taenia spp. infection. Consumption of raw vegetables attributed to $74 \%$ of $T$. solium cysticerci exposure-positive cases and consumption of undercooked beef attributed to $77 \%$ of taeniasis cases in these communities.

Conclusions: The prevalence of T. solium seroexposure in Dak Lak is consistent with those reported in other regions of Vietnam. The identified risk factors associated with the prevalence of $T$. solium seroexposure and taeniasis infection in Dak Lak are modifiable and thus advocate for targeted community intervention programs to mitigating these risks.
\end{abstract}

Keywords: Epidemiology, Taeniasis, Cysticercosis, Risk factors, Vietnam

\section{Background}

Taenia solium cysticercosis and human taeniasis are considered neglected tropical diseases by the World Health Organization [1]. When accidentally ingested either directly, or indirectly via contaminated food and water, eggs of $T$. solium hatch and lodge in the skeletal

\footnotetext{
* Correspondence: ngocn4@student.unimelb.edu.au; theeveret@gmail.com

${ }^{1}$ Faculty of Veterinary and Agricultural Sciences, University of Melbourne,

Parkville, VIC 3052, Australia

${ }^{2}$ Faculty of Animal Sciences and Veterinary Medicine, Tay Nguyen University, Dak Lak, Vietnam

Full list of author information is available at the end of the article
}

muscle, under the skin and/or within the central nervous system causing muscular-, subcutaneous- and/or neuro-cysticercosis. Neurocysticercosis is a common cause of epilepsy, syncope, paralysis and chronic headache $[2,3]$. Globally, the disease has been estimated to cause approximately 2.8 million DALYs (disability-adjusted life-years) lost and between 2010 and 2015 approximately 300,000 individuals were estimated to be infected globally, resulting in over 28,100 deaths [4]. Consumption of raw or undercooked meat and/or visceral organs containing cysticerci of T. solium, Taenia

(c) The Author(s). 2018 Open Access This article is distributed under the terms of the Creative Commons Attribution 4.0 International License (http://creativecommons.org/licenses/by/4.0/), which permits unrestricted use, distribution, and reproduction in any medium, provided you give appropriate credit to the original author(s) and the source, provide a link to the Creative Commons license, and indicate if changes were made. The Creative Commons Public Domain Dedication waiver (http://creativecommons.org/publicdomain/zero/1.0/) applies to the data made available in this article, unless otherwise stated. 
saginata or Taenia asiatica cause taeniasis. Taeniasis reduces the quality of life, is responsible for diagnostic and treatment costs in infected individuals and indirectly contributes to poverty due to losses in livestock production arising from organ condemnation and loss of market access [5].

Vietnam is considered endemic for taeniasis and $T$. solium cysticercosis [6, 7]. Previous research focusing on these diseases in Vietnam has mostly been carried out in the north of the country. A review of the literature shows that common risk factors for taeniasis and cysticercosis include consumption of raw or undercooked meat and vegetables, lack of functioning latrines, husbandry practices allowing pigs to free-roam and routine use of human waste for fertilizing and irrigating crops [8-12]. Information on the prevalence of and risk factors for taeniasis and $T$. solium cysticercosis in the central and southern areas of Vietnam is scarce and outdated [12]. In Dak Lak province, there is no information available on the infection status of $T$. saginata in livestock, however our research data on the seroprevalence of cysticercosis in pigs was $0.95 \%$ [13]. A previous study carried out by our group using a newly developed multiplex real-time PCR found the prevalence of Taenia solium, T. saginata and $T$. asiatica taeniasis to be $1.2 \%, 5.8 \%$ and $1.5 \%$, respectively in Dak Lak province [14]. In this study, our aims were to: i) estimate the seroprevalence of $T$. solium cysticerci exposure using the lentil-lectin purified glycoprotein enzyme-linked immunoelectrotransfer blot (LLGP-EITB) assay; ii) identify and quantify risk factors for human $T$. solium cysticerci exposure and taeniasis (Taenia spp. infection), that inform optimal risk mitigation measures in studied communities in Dak Lak province, Vietnam.

\section{Methods}

\section{Studying site and sampling}

The study was deployed in the districts of Buon Don, Krong Nang and M'Drak in the province of Dak Lak in the Central Highlands of Vietnam between May and October 2015. M'Drak is located in the east of Dak Lak province with an average altitude of $400 \mathrm{~m}$ to $500 \mathrm{~m}$ and has a tropical monsoon climate typical of the Vietnam's Central Coast. Krong Nang is situated in the north of the province at an altitude of $800 \mathrm{~m}$. Buon Don, situated to the west of the province with an average elevation of $330 \mathrm{~m}$ and has a hot and dry climate. The standard of living in this area of Vietnam is generally poor. The literacy rate among local residents aged between 15 and 60 in rural areas was low [15]. Open defaecation using outdoor pit latrines is common practice and livestock access to these latrine areas is usually unrestricted. The practice of non-confinement of pigs and cattle is common with slaughter activities often carried out in backyards
[12]. The sample size was calculated based on the previous study of Van De at al. [31]. The seroprevalence of $T$. solium cysticerci exposure was assumed to be $7 \%$ and there was 95\% certainty that this estimate was within 5\% of the true population value (i.e. the prevalence ranged from $2 \%$ to $12 \%)$. Ignoring the tendency for $T$. solium cysticerci exposure to cluster within households we estimated that a total of 100 samples were required. We then assumed the average number of individuals eligible for the study per household was three and the between household (cluster) variance in seroprevalence was around 1.14 times greater than the within household (cluster) variance, returning an intra-class correlation coefficient of 0.07 [16]. Our revised sample size, accounting for the tendency of cluster within households, was 114 for each district and 342 for the whole province

A sampling frame listing the name of all villages in Dak Lak province was obtained from the Sub-Department of Animal Health office within the Ministry of Agriculture. Villages eligible for sampling comprised those with more than 1000 pigs, as recorded by the Sub-Department of Animal Health. All eligible villages within each district were assigned a number and two numbers chosen at random to select villages from each district for inclusion in the study. A list of householder names within each selected village was obtained from each village head person, and household names were assigned a numeric code. The sheet of paper of numeric household codes for each village were cut into pieces and placed face-down on a table. The village head person was asked to select 50 households at random. Each village has a number of households between 200 and 300. All selected households were visited several days before the proposed sampling date to obtain consent from participants to take part in the study. From each household between one and five individuals were requested to take part in the study. Individuals eligible for inclusion in the study were healthy, not pregnant and over seven years of age. Individuals requested to take part in the study signed a consent form. Those that were under 18 years of age were required to provide written consent as well as written consent from either their parents or legal guardians. At the time of consent, each study participant was given a labeled stool container, with instructions that the container would be collected on the date of sampling, several days later. Five $\mathrm{mL}$ of venous blood was collected into plain clotting tubes from consenting study participants by local medical staff. The blood was kept at ambient temperature for clotting and then centrifuged at $3200 \times \mathrm{g}$ for $5 \mathrm{~min}$ to collect serum. The serum was stored at $-20{ }^{\circ} \mathrm{C}$ until use.

\section{Household questionnaire}

Each study participant was requested to answer a prepared questionnaire (Additional File 1). The questionnaire 
comprised of two sections. The first section collected information at the household level and was completed by the nominated head of the household. Questions included in this section solicited details on housing type, details of the type of latrines in use (if any), the presence and type of backyard pigsties, the source(s) of drinking water and activities associated with cultivation, irrigation and fertilization of home or farm grown vegetables. The second part of the questionnaire solicited details at the individual study participant level including their age, gender, the highest level of education achieved, dietary practices, and hygiene. All questionnaires were conducted in local Vietnamese phraseology and the validity of the questionnaire was pre-tested on thirty individuals in another community in Dak Lak province before application to the field survey. In addition, interviewers were trained before administering the questionnaires.

\section{Laboratory procedures}

\section{Detection of antibodies against T. solium cysticerci}

Serum samples were subjected to LLGP-EITB assay to detect antibodies against $T$. solium cysticerci. This assay has a diagnostic sensitivity of $98 \%$ and a diagnostic specificity of $100 \%$. The assay was performed as previously described by Tsang et al. (1989) [17] and Noh et al. (2014) [18].

\section{Detection of Taenia spp. infection}

A total of 342 faecal samples were subject to a multiplex real-time PCR (T3qPCR) to detect all three species of Taenia, notably, T. solium, Taenia saginata and Taenia asiatica. The assay has been reported to have a diagnostic sensitivity of $94 \%$ and a diagnostic specificity of $98 \%$. The positive results of T3qPCR were confirmed using either a conventional multiplex PCR targeting T. solium, T. saginata and T. asiatica [19] or a singleplex PCR for Taenia sp. following DNA sequencing. The methods and results have been described and published elsewhere [14].

\section{Statistical methods}

Risk factors for T. solium cysticerci exposure and taeniasis (Taenia spp. infection) in humans in the communities of Dak Lak province were identified using logistic regression. The outcome of interest for this study was a binary response variable, $\mathrm{Y}_{\mathrm{i}}$, taking the value 1 if an individual was $T$. solium cysticerci exposure-positive using the LLGP-EITB or in the case of taeniasis, was positive for Taenia spp. infection by T3qPCR, and 0 otherwise.

This study did not use a simple random sampling design to avoid erroneous point estimates of the strength of the association between hypothesized risk factors and the outcome of interest and their standard errors. The data were analyzed in a manner that respected the three-stage cluster design (villages within districts formed the primary sampling units, households within villages formed the secondary sampling units and individuals within households formed the tertiary sampling units). Sampling weights $W_{I}$, provided an estimate of the inverse probability of an individual being selected for study (Lumley 2010) [20] and were quantified for each study participant as follows:

$$
w_{1}=\frac{V}{v} x \frac{H}{h} x \frac{M}{m}
$$

In Eq. $1, V$ and $v$ represent the total number of villages in each district and the number of sampled villages in each district, respectively; $H$ and $h$ represent the total number of households in each sampled village and the number of sampled households in each village, respectively; and $M$ and $m$ represent the total number of individuals in each sampled household, and the number of sampled individuals in each household, respectively.

Unconditional associations between each of the hypothesized risk factors from the questionnaire and the outcome of interest were computed using the odds ratio. Hypothesized risk factors (explanatory variables) with unconditional associations that were significant at $P<0.20$ level (2-sided) were selected for multivariable modeling.

A fixed-effects logistic regression model was developed where the probability of an individual being Taenia- or T. solium cysticerci exposure-positive was parameterized as a function of the explanatory variables with unconditional associations significant at $\mathrm{P}<0.20$, as described above. Explanatory variables that were not statistically significant were removed from the model one at a time, beginning with the least significant, until the estimated regression coefficients for the explanatory variables retained were significant at an alpha level of less than 0.05 . Explanatory variables that were excluded at the initial screening stage were tested for inclusion in the final model and were retained if their inclusion changed any of the estimated regression coefficients by more than $20 \%$. Biologically plausible two-way interactions were tested and none were significant at an alpha level of 0.05 .

To account for the hierarchical structure of the data, that is, villages within districts and households within villages and study participants within households, we extended the model to include village- and household-level random effect terms using the lme4 package [21] in $R$ version 3.4.3 [22]. This extension to the model was informative because it provided the opportunity to distinguish the influence of unobserved explanatory variables operating at the village, household and individual level on Taenia- and T. solium cysticerci exposure-positive risk. In our multilevel model the variance of the random effect terms at the village and household level were negligible, and the point estimates of the regression coefficients and standard errors for the explanatory variables were identical to the fixed-effects model. For parsimony, 
we report the results of the fixed-effects regression model adjusted for the three-stage sampling design using the survey package [23] in R.

The contribution of each of the explanatory variables in the final model on Taenia- and T. solium cysticerci exposure-positive risk was quantified using the population attributable fraction. The population attributable fraction for a given explanatory variable is the proportional reduction in outcome event incidence expected by either eliminating exposure to the variable or completely preventing the effects of exposure, assuming the explanatory variable is causative and assuming there has been no bias and sampling error in the study population [24]. Using the method of Toschke et al. (2007) [25], adjusted population attributable fractions were calculated for each of the explanatory variables that were significant in the mixed-effects logistic regression model.

\section{Results}

General description of study population and data structure Table 1 presents the structure of the data. In total 342 individuals, from 190 households in six villages in three districts of Dak Lak province consented to take part in the study. A household had, on average, two individuals that consented to take part (minimum 1; maximum 5). In total, only $25 \%$ (95\% confidence interval (CI) $19 \%$ to $32 \%, 47$ of 190) of households possessed a latrine and $25 \%$ (95\% CI $19 \%$ to $32 \%, 48$ of 190 ) of households sourced their water for daily activities, including drinking, from lakes, streams or ponds. Untreated drinking water was consumed by $60 \%$ ( $95 \%$ CI $53 \%$ to $67 \%, 115$ of 190) of households (Table 2). The literacy rate among participants was $56 \%$ (95\% CI $51 \%$ to $62 \%, 193$ of 342 ). Most participants (87\%, 95\% CI $80 \%$ to $88 \%$ ) listed farming as their main occupational activity (Table 3 ). The percentage of study participants that did not wash their hands after defaecation or before eating was 35\% (95\% CI $31 \%$ to $41 \%, 121$ of 339$)$ and $44 \%$ (95\% CI $39 \%$ to $50 \%, 150$ of 339), respectively.

Total of 342 serum samples collected from study participants, three were excluded due to hemolysis resulting in 339 samples included for further analysis. Of 339

Table 1 Structure of the data from 342 study participants from six villages in M'Drak, Buon Don and Krong Nang districts

\begin{tabular}{llll}
\hline Level & Number & \multicolumn{2}{l}{ Number at the next highest level } \\
\cline { 3 - 4 } & & Mean & Range \\
\hline Districts & 3 & - & - \\
Villages & 6 & 2 & 2 \\
Households & 190 & 31 & 18 to 40 \\
Humans $^{\mathrm{a}}$ & 342 & 2 & 1 to 5 \\
\hline
\end{tabular}

${ }^{a} A$ total of 342 individuals participated in this study. The mean of individuals per household was 2 (range 1 to 5 )
Table 2 General description of household data

\begin{tabular}{|c|c|c|}
\hline Characteristic & Frequency & Percentage $(95 \% \mathrm{Cl})$ \\
\hline Number of households & 190 & \\
\hline \multicolumn{3}{|l|}{ District } \\
\hline M'Drak & 58 & 30 (24 to 38$)$ \\
\hline Buon Don & 66 & 35 (28 to 42$)$ \\
\hline Krong Nang & 66 & 35 (28 to 42 ) \\
\hline \multicolumn{3}{|l|}{ Owning pigs } \\
\hline Yes & 37 & 19 (14 to 26$)$ \\
\hline No & 153 & 81 (74 to 86$)$ \\
\hline \multicolumn{3}{|l|}{ Presence of latrine } \\
\hline Yes & 47 & 25 (19 to 32$)$ \\
\hline No & 143 & 75 (68 to 81$)$ \\
\hline \multicolumn{3}{|l|}{ Source of drinking water } \\
\hline Pipe/well/rain water & 142 & 74 (66 to 79$)$ \\
\hline Lake/stream/pond & 48 & 26 (19 to 32$)$ \\
\hline \multicolumn{3}{|l|}{ Water treatment } \\
\hline Yes & 75 & 39 (32 to 47$)$ \\
\hline No & 115 & 60 (53 to 67$)$ \\
\hline \multicolumn{3}{|l|}{ Are vegetables fertilized? } \\
\hline Yes & 81 & 43 (35 to 50$)$ \\
\hline No & 109 & 57 (50 to 64) \\
\hline
\end{tabular}

Table 3 Demographic data for individual study participants

\begin{tabular}{|c|c|c|}
\hline Characteristic & Frequency & Percentage $(95 \% \mathrm{Cl})$ \\
\hline Total number of participants & 342 & \\
\hline \multicolumn{3}{|l|}{ Age (years) } \\
\hline$<25$ & 63 & 18 (14 to 23$)$ \\
\hline $25-60$ & 223 & 65 (60 to 70$)$ \\
\hline$>60$ & 56 & 16 (13 to 21$)$ \\
\hline \multicolumn{3}{|l|}{ Sex } \\
\hline Male & 104 & 30 (26 to 36$)$ \\
\hline Female & 238 & 70 (64 to 74$)$ \\
\hline \multicolumn{3}{|l|}{ Highest level of education } \\
\hline None & 149 & 44 (38 to 49 ) \\
\hline Primary/secondary & 184 & 54 (48 to 59) \\
\hline Tertiary & 9 & $2.6(1.3$ to 5.1$)$ \\
\hline \multicolumn{3}{|l|}{ Primary activity } \\
\hline Farming & 298 & 87 (83 to 90$)$ \\
\hline Livestock & 14 & 4.1 (2.3 to 6.9$)$ \\
\hline Civil service & 5 & 1.5 (0.5 to 3.6$)$ \\
\hline Other & 25 & $7.3(4.9$ to 11$)$ \\
\hline \multicolumn{3}{|l|}{ Ethnicity } \\
\hline Kinh & 40 & 11 (8.6 to 15$)$ \\
\hline Ede & 302 & 88 (84 to 91) \\
\hline
\end{tabular}


serum samples, antibodies against $T$. solium cysticerci were identified in 17 individuals (5\%, 95\% CI $3 \%$ to $8 \%$ ). Among 342 faecal samples, 23 (6.7\%, 95\% CI 4.4\% to 10\%) were positive for Taenia spp. Single infection of $T$. solium and T. saginata were identified in three and 14 samples, respectively; mixed infections of T. solium and $T$. saginata occurred in one samples, and of $T$. saginata and T. asiatica in five samples [14].

\section{Risk factors for $T$. solium cysticerci exposure}

Of the data recorded using the questionnaire, we identified three factors associated with an individual's likelihood of being exposure-positive to $T$. solium cysticerci based on the results of the LLGP-EITB assay. These were, the frequent consumption of raw vegetables, sourcing drinking water from lakes, streams or ponds and outdoor defaecation.

Estimated regression coefficients for consumption of raw vegetables, the source of drinking water and routine location of defaecation are provided in Table 4. After adjusting for other explanatory variables in the model, the odds of an individual who often consumed raw vegetables being $T$. solium cysticerci exposure-positive was 9.98 (95\% CI 2.89 to 34.4) times that of an individual who consumed raw vegetables rarely. The odds ratio for those who routinely defaecated outdoors was similar, at 11.1 (95\% CI 2.97 to 42.0 ). The adjusted population attributable fraction for the consumption of raw vegetables was $74 \%$ and thus, reducing the prevalence of raw vegetable consumption in the population is expected to reduce the exposure to T. solium cysticerci by a factor of $74 \%$. Similarly, the population attributable fraction estimate for routine location of defaecation was $60 \%$.

The odds of $T$. solium cysticerci exposure for those that routinely sourced their drinking water from streams, ponds or lakes was 3.94 (95\% CI 1.29 to 11.9$)$ times higher than the odds of $T$. solium cysticerci exposure for those that sourced their water from wells.

\section{Risk factors for Taenia spp. infection}

We identified five factors associated with an individual's likelihood of infection with Taenia spp. gender, the frequent consumption of pork tongue, the frequent consumption of undercooked pork, frequent consumption of undercooked beef, and observation of Taenia proglottids shedding from either themselves, their relatives or neighbors (Table 5).

After adjusting for other explanatory variables in the model, the odds of Taenia spp. infection for an individual who frequently consumed pork tongue was 4.62 (95\% CI 1.49 to 14.3 ) times the odds for an individual who consumed pork tongue only rarely. Males had higher odds of being Taenia spp. positive compared with females (OR 2.77, 95\% CI 1.00 to 7.68).

Among the modifiable risk factors for Taenia spp. infection identified in this study, the adjusted population attributable fraction for frequent consumption of undercooked beef was $77 \%$. The population attributable fractions for frequent consumption of undercooked pork and pork tongue were $24 \%$ and $26 \%$, respectively. Assuming undercooked beef consumption is a component cause of taeniasis risk, reducing the prevalence of this practice in the population is expected to reduce the overall prevalence of taeniasis by a factor of $77 \%$.

\section{Discussion}

The seroprevalence of exposure to T. solium in rural communities in Dak Lak in the Central Highlands was $5 \%$ (95\% CI $3 \%$ to $8 \%$ ). This prevalence is relatively similar to other regions across Vietnam. Phan et al. (2001) [11] reported a $4 \%$ seroprevalence of cysticercosis across 22 provinces in the south of Vietnam. For studies in the north and northwest, where T. solium is reported to be endemic, the seroprevalence ranged from 2 to $6 \%$ [2629]. In this study, various risk factors were evaluated to

Table 4 Risk factors associated with T. solium cysticerci exposure identified by a LLGP-EITB assay

\begin{tabular}{|c|c|c|c|c|c|}
\hline Explanatory variable & Number positive & Number of individuals & Regression coefficient (SE) & $P$ value & Adjusted OR (95\% Cl) \\
\hline Intercept & 17 & 339 & $-6.4694(1.0615)$ & $<0.01$ & - \\
\hline \multicolumn{4}{|c|}{ Raw vegetable consumption } & $<0.01$ & \\
\hline Occasionally & 4 & 226 & Reference & & 1.00 \\
\hline Often & 13 & 113 & $2.3006(0.6318)$ & & $9.98(2.89 \text { to } 34.4)^{a}$ \\
\hline \multicolumn{4}{|l|}{ Area of defaecation ${ }^{\mathrm{b}}$} & $<0.01$ & \\
\hline Close latrine & 5 & 228 & Reference & & 1.00 \\
\hline Outdoor & 12 & 110 & $2.4125(0.6763)$ & & 11.1 (2.97 to 42.0$)$ \\
\hline \multicolumn{4}{|c|}{ Source of drinking water } & $<0.05$ & \\
\hline Other ${ }^{c}$ & 9 & 253 & Reference & & 1.00 \\
\hline Stream/Lake/Pond & 8 & 86 & $1.3702(0.5684)$ & & 3.94 (1.29 to 11.9$)$ \\
\hline
\end{tabular}

Interpretation: After adjusting for other explanatory variables in the model, the odds of an individual who consumed raw vegetables often being $T$. solium cysticerci exposure was 9.98 ( $95 \% \mathrm{Cl} 2.89$ to 34.4$)$ times that of an individual who consumed rare vegetables rarely

${ }^{\mathrm{b}} \mathrm{A}$ single individual with a missing value for area of defaecation excluded

'Drinking water sourced from wells, pipes, rainy and packed water 
Table 5 Logistic regression model showing the effect of sex, dietary and observation of proglottids on the odds of a taeniasis case

\begin{tabular}{|c|c|c|c|c|c|}
\hline Explanatory variable & Number positive & Number of individuals & Regression coefficient (SE) & $P$ value & Adjusted OR (95\% Cl) \\
\hline Intercept & 23 & 342 & $-6.6069(1.0365)$ & $<0.01$ & - \\
\hline \multicolumn{4}{|l|}{ Sex: } & $<0.05$ & \\
\hline Female & 8 & 238 & Reference & & 1.00 \\
\hline Male & 15 & 104 & $1.0183(0.5209)$ & & $2.77(1.00 \text { to } 7.68)^{a}$ \\
\hline \multicolumn{4}{|c|}{ Undercooked pork consumed: } & $<0.05$ & \\
\hline No & 9 & 241 & Reference & & 1.00 \\
\hline Yes & 14 & 101 & $1.3023(0.5695)$ & & $3.68(1.20$ to 11.2$)$ \\
\hline \multicolumn{4}{|c|}{ Pork tongue consumed } & $<0.01$ & \\
\hline Rare & 15 & 305 & Reference & & 1.00 \\
\hline Often & 8 & 37 & $1.5309(0.5778)$ & & $4.62(1.49$ to 14.3$)$ \\
\hline \multicolumn{4}{|c|}{ Undercooked beef consumed } & $<0.01$ & \\
\hline No & 2 & 154 & Reference & & 1.00 \\
\hline Yes & 21 & 188 & $1.935(0.8070)$ & & $6.92(1.42$ to 33.7$)$ \\
\hline \multicolumn{4}{|l|}{ Proglottids observed } & $<0.01$ & \\
\hline No & 12 & 302 & Reference & & 1.00 \\
\hline Yes & 11 & 40 & $2.592(0.5842)$ & & 13.36 (4.25 to 41.9 ) \\
\hline
\end{tabular}

determine their association with seroexposure to $T$. solium using the LLGP-EITB assay. Of these, raw vegetable consumption, the source of drinking water and routine location of defaecation were significantly associated with an increase in T. solium cysticerci exposure (Table 4). It is known that risk factors for transmission and circulation of $T$. solium cysticercosis are numerous and may vary in different settings. The identified risk factors in Dak Lak were consistent with that of study carried out in the north and south of Vietnam [11, 30]. In contrast to studies in Vietnam's north, the utilisation of night-soil was not associated with the increase in $T$. solium exposure observed in these study communities $[12,31]$. Similar to the studies in western Sichuan (China) [32], Mbeya (Tanzania) [26] and northern India [33], routine open defaecation, utilisation of unsafe water sources and consumption of raw vegetables significantly increased the risk of cysticercosis in Dak Lak. However, Cherian et al. (2014) [34] reported that these factors were not contributive in individuals who had epilepsy in Kerala, India. Research on the epidemiological characteristics of human T. solium cysticercosis in Nigeria [35], Tanzania [36], and Guatemala [37] found that gender and age [38] were associated with cysticercosis seropositivity, in which the female gender and increased age were significantly linked to the prevalence of $T$. solium. In our study, age and gender did not contribute to seroexposure of $T$. solium cysticerci.

Each of the identified risk factors in this study are eminently modifiable and if effective interventions are deployed, we predict that there will be marked reduction (in the order of $60 \%$ to $74 \%$ ) in the prevalence of exposure to $T$. solium cysticerci in this area of Vietnam. Dak Lak province has a population of approximately 1.8 million [39]; the survey adjusted apparent prevalence of exposure to T. solium cysticerci, as estimated in this study, at $5.01 \%$ equates to approximately 90,000 exposure-positive individuals. With a population attributable fraction for routine location of defaecation at $60 \%$, and assuming an intervention program to educate inhabitants to practice defaecation indoors, we estimate that the number of exposure-positive individuals will be reduced to approximately 36,000 individuals if the program is $100 \%$ successful. Even with $50 \%$ efficacy of the intervention program, we estimate the number of exposurepositive individuals would be in the order of 63,000 , a substantial reduction from the current prevalence estimate of 90,000. The expected savings to the public health sector (in terms of avoided diagnostics and treatments) is likely to be substantial. If both location of defaecation and raw vegetable consumption practices are modified concurrently, the expected population attributable fraction is $89 \%$. Outdoor defaecation results in the contamination of the environment including water and vegetables; thus, to reduce or mitigate the burden of T. solium cysticerci exposure of inhabitants in the communities of Dak Lak province, this factor should be given the highest priority of implementation as an intervention program.

Taenia spp. infection risk in in this study was associated with gender, the consumption of pork tongue, consumption of undercooked pork and beef, and observation of 
Taenia proglottids shedding from either themselves, their relatives or neighbors (Table 5). Among these factors, the consumption of pork tongue, and consumption of undercooked pork and beef are modifiable. Consumption of undercooked beef had the highest population attributable fraction of $77 \%$ compared to other factors which ranged from 24 to $26 \%$. Thus, an intervention program aimed at discouraging practices of consuming undercooked beef could potentially result in a 77\% reduction in the number of cases of taeniasis in the region. The strong association of undercooked beef consumption with Taenia positivity could explain the dominant prevalence of $T$. saginata in this study population at $5.8 \%$ compared to $1.2 \%$ with $T$. solium and $1.5 \%$ with $T$. asiatica [14], because beef containing $T$. saginata cysticerci is the cause of $T$. saginata taeniasis in humans.

Males had a higher likelihood of taeniasis compared to females. In rural communities of Dak Lak, males commonly consume undercooked beef, pork, or pork visceral organs while drinking alcohol as part of traditional cultural practices. Thus, educating the community on appropriate consumption of meat, targeting male members of the community is expected to have a substantial impact on the prevalence of taeniasis, although altering cultural dietary practices is recognized as a challenging approach to successful intervention. The most notable limitation of this study was the low prevalence of infection with individual species of Taenia, namely, T. solium, $T$. saginata and $T$. asiatica in study communities. This deterred our ability to build a model to predict risk factors associated with species-level Taenia infection in this study.

A three-stage cluster sampling design was used for this study with villages within districts as the primary sampling unit, households within villages as the secondary sampling unit and individuals within households as the tertiary sampling unit. Given the hierarchical structure of our data it was our a priori belief that observations made on individual study participants were not likely to be independent, justifying the use of regression modelling approaches that accounted for this non-independence. In our mixed-effects logistic regression model the variance of the random effect terms at both the village and individual household levels were negligible. Our inference is that in this population most of the unmeasured Taenia/cysticerci-exposure risks resided at the individual (as opposed to either the village or household) level. The practical implications of this finding are that in addition to addressing the modifiable risk factors identified in these analyses, health education programs need to be focused at the individual level.

Since many of the identified risk factors are traditional/behavioural practices in this area of Vietnam, the probability to induce permanent behavioural change from a single education campaign is likely to be small. A One Health approach utilising the expertise of anthropologists might be one way of effecting permanent behavioural change. Long term behavioural change in a population and on-going political support for health interventions occur when aspects of health risk, the economic benefits of disease control and the economic costs associated with disease can be quantified [40]. Financial and human resources are critical factors influencing the success of disease intervention programs. If resources are not available to support an integrated, long-term control and eradication program, a selective approach provides a suitable alternative with the selection of those interventions that provide the most cost-effective return on investment being preferred [41, 42]. The resource for taeniasis, cysticercosis intervention programs in Dak Lak province is limited; therefore, a selective approach is necessary. As discussed above, health education programs involving both the human medical and veterinary health sectors are a feasible intervention option. Choosing a small number of risk factors that make the greatest contribution to the prevalence of taeniasis or cysticercosis are logical targets within an education campaign, for example, indoor latrine construction will ultimately prove the most cost-effective approach.

\section{Conclusions}

The prevalence of seroexposure to T. solium in the communities of Dak Lak is consistent with that of other regions in Vietnam. Identified risk factors are modifiable, and associated strongly with the prevalence of T. solium seroexposure and Taenia spp. infection in Dak Lak communities, thus advocating for targeted community intervention programs in mitigating these risks.

\section{Additional file}

Additional file 1: Questionnaire 1 A blank copy of the questionnaire used in this study. (DOCX $178 \mathrm{~kb}$ )

\section{Abbreviations}

Cl: Confidence interval; LLGP-EITB: Lentil lectin purified glycoprotein enzymelinked immunoelectrotransfer blot assay; OR: Odds ratio; T3qPCR: Multiplex real-time PCR

\section{Acknowledgements \\ We are grateful to the Institute of Biotechnology and Environment Tay Nguyen University for providing resources and facilities for the fieldwork. We thank Drs. John Noh and Sukwan Handali from the Center for Diseases Control and Prevention, GA, USA for EITB analysis. The Authors are grateful to village medical staff at Buon Don, Krong Nang and M'Drak district for assisting with sample collection. The authors are most thankful to Assoc. Prof. Than Trong Quang, Ms. Nguyen Thi Ngoc Hien, Ms. Long Khanh Linh, and Ms. Nguyen Thi Lan Huong, who assisted with laboratory work and fieldwork.}

\section{Funding}

This research was self-funded by RJT. This work was done with partial support for travel from the Faculty of Veterinary and Agricultural Sciences, University of Melbourne, Australia. DNN received PhD scholarship from Australia Awards Scholarships, Department of Foreign Affairs and Trade, Australia Government. 


\section{Availability of data and materials}

Supporting data and materials are available from the corresponding author anytime upon reasonable request.

\section{Authors' contributions}

DNN designed study, analyzed data and wrote manuscript; MAS: assisted with analyses of data and edited paper; KB, TVP, VTN and TV: assisted laboratory work and fieldwork; RJT provided material, supervised study and edited paper. All authors read and approved the final manuscript.

\section{Ethics approval and consent to participate}

This study was reviewed and approved by The University of Melbourne Human Research Ethics Committee through (Ethics ID numer 1,443,512), and conducted under the supervision of the local Center for Public Health, Dak Lak, Vietnam. Informed and voluntary consent of each participant (or guardian) was obtained prior to their participation and inclusion in the research project through a Plain Language Statement and the participants consent established by use of a Consent Form.

\section{Consent for publication}

Not applicable.

\section{Competing interests}

The authors declare that they have no competing interests.

\section{Publisher's Note}

Springer Nature remains neutral with regard to jurisdictional claims in published maps and institutional affiliations.

\footnotetext{
Author details

${ }^{1}$ Faculty of Veterinary and Agricultural Sciences, University of Melbourne, Parkville, VIC 3052, Australia. ${ }^{2}$ Faculty of Animal Sciences and Veterinary Medicine, Tay Nguyen University, Dak Lak, Vietnam. ${ }^{3}$ Department of Livestock, Montana Veterinary Diagnostic Lab, Bozeman, MT, USA. ${ }^{4}$ Faculty of Medicine and Pharmacy, Tay Nguyen University, Dak Lak, Vietnam. ${ }^{5}$ Department of Physiology, Pathology and Immunology, Pham Ngoc Thach University of Medicine, Ho Chi Minh, Vietnam.
}

Received: 24 June 2018 Accepted: 4 October 2018 Published online: 22 October 2018

\section{References}

1. FAONHO. Multicriterial-based Ranking for Risk Management of Food-borne Parasites. Microbiological risk assessment series no 23. Rome: WHO press; 2014.

2. Ndimubanzi PC, Carabin H, Budke CM, Nguyen H, Qian YJ, Rainwater E, et al A systematic review of the frequency of neurocyticercosis with a focus on people with epilepsy. PLoS Negl Trop Dis. 2010;4:e870.

3. Garcia HH, Nash TE, Del Brutto $\mathrm{OH}$. Clinical symptoms, diagnosis, and treatment of neurocysticercosis. Lancet Neurol. 2014;13:1202-15.

4. Torgerson PR, Devleesschauwer B, Praet N, Speybroeck N, Willingham AL, Kasuga F, et al. World Health Organization estimates of the global and regional disease burden of 11 foodborne parasitic diseases, 2010: a data synthesis. PLoS Med. 2015;12:e1001920.

5. WHO. Taeniasis/cysticercosis. WHO. 2018. http://www.who.int/news-room/ fact-sheets/detail/taeniasis-cysticercosis. Accessed 22 Feb 2018.

6. Aung AK, Spelman DW. Taenia solium taeniasis and cysticercosis in Southeast Asia. Am J Trop Med Hyg. 2016;94:947-54.

7. Wu HW, Ito A, Ai L, Zhou XN, Acosta LP, Lee WA. Cysticercosis/taeniasis endemicity in Southeast Asia: current status and control measures. Acta Trop. 2015;165:121-32.

8. NIMPE. Taeniasis and Cysticercosis. In: Review workshop on helminthiasis control activities in the period 2006-2011 and implementing the workplan for the period 2012-2015. Ministry of Health of Vietnam; 2012.

9. Vien HV, Hung NM, Thach DTC, Thuan LK, Dung DT, Hop NT, et al. Investigate Taenia infection in two communes of Tan Son district, Phu Tho province. J Malar Parasite Dis Control. 2012;1:69-74.

10. Van Tuan B, Van CN. Situation of Taenia saginata infestation and effectiveness of some control measures in Dak Mon commune (Dak Glei) and Yaxier commune (Sa Thay), Kon Tum province. J Malar Parasite Dis Control. 2014;1:41-8.
11. Anh Tuan P, Dung TTK, Nhi VA. Sero-epidemiological investigation of cysticercosis in the southern provinces. J Malar Parasite Dis Control. 2001;4:81-7.

12. Ng-Nguyen D, Stevenson MA, Traub RJ. A systematic review of taeniasis, cysticercosis and trichinellosis in Vietnam. Parasit Vectors. 2017;10:150.

13. Ng-nguyen D, Noh J, Breen K, Stevenson MA, Handali S, Traub RJ. The epidemiology of porcine Taenia solium cysticercosis in communities of the Central Highlands in Vietnam. Parasit Vectors. 2018;11:360.

14. Ng-Nguyen D, Stevenson MA, Dorny P, Gabriël S, Van VT, Nguyen VT, et al. Comparison of a new multiplex real-time PCR with the Kato Katz thick smear and copro-antigen ELISA for the detection and differentiation of Taenia spp. in human stools. PLoS Negl Trop Dis. 2017;11:e0005743.

15. General statistics office of Viet Nam. Percentage of literate population at 15 years of age and above by province. 2016. https://www.gso.gov.vn/ default_en.aspx?tabid=774. Accessed 8 Jan 2018.

16. Lucas LA, Phillip MK, Peter BG, Mark ON, Gerald M, Esther S. A survey of bovine cysticercosis/human taeniosis in Northern Turkana District, Kenya. Preventive Veterinary Medicine. 2009;89(3-4):197-204.

17. Tsang VCW, Brand JA, Boyer AE. An enzyme-linked immunoelectrotransfer blot assay and glycoprotein antigens for diagnosing human cysticercosis (Taenia solium). J Infect Dis. 1989;159:50-9.

18. Noh J, Rodriguez S, Lee YM, Handali S, Gonzalez AE, Gilman RH, et al. Recombinant protein- and synthetic peptide-based immunoblot test for diagnosis of neurocysticercosis. J Clin Microbiol. 2014;52:1429-34.

19. Jeon HK, Chai JY, Kong Y, Waikagul J, Insisiengmay B, Rim HJ, et al. Differential diagnosis of Taenia asiatica using multiplex PCR. Exp Parasitol. 2009:121:151-6.

20. Lumley T. Complex surveys a guide to analysis using R. New York: Wiley; 2010.

21. Bates $D$, Machler M, Bolker B, Walker S. Fitting linear mixed-effects models using Ime4. J Stat Softw. 2015;67:1-48.

22. Team R Core. R: A Language and Environment for Statistical Computing. 2017. http://www.r-project.org.

23. Lumley T. Analysis of complex survey samples. J Stat Softw. 2004:9:1-19.

24. Whittemore AS. Statistical methods for estimating attributable risk from retrospective data. Stat Med. 1982:1:229-43.

25. Toschke AM, Rückinger S, Böhler E, Von Kries R. Adjusted population attributable fractions and preventable potential of risk factors for childhood obesity. Public Health Nutr. 2007;10:902-6.

26. Trung DD, Praet N, Cam TDT, Lam BVT, Manh HN, Gabriël S, et al. Assessing the burden of human cysticercosis in Vietnam. Trop Med Int Heal. 2013;18:352-6.

27. Somers R, Dorny P, Nguyen VK, Dang TCT, Goddeeris B, Craig PS, et al. Taenia solium taeniasis and cysticercosis in three communities in North Vietnam. Trop Med Int Heal. 2006;11:65-72.

28. Doanh NQ, Kim NT, De NV, Lung NL. Result of survey on taeniasis and cysticercosis humans and pigs in Bac Ninh and Bac Kan provinces. Vet Sci Tech. 2002;9:46-9.

29. Erhart A, Dorny P, Van De N, Vien HV, Thach DC, Toan ND, et al. Taenia solium cysticercosis in a village in northern Viet Nam: Sero-prevalence study using an ELISA for detecting circulating antigen. Trans R Soc Trop Med Hyg 2002;96:270-2.

30. Ho ST. Study on genotype of pathogen, clinical, subclinical symptoms, treatment efficacy for taeniasis and cysticercosis patients in National Institute of Malariology, Parasitology and Entomology 2007-2010: Hano Medical University; 2013. http://uanan.nlv.gov.vn/luanan?a=d\&d= TTcFqWrCyuSa2013.1.4\&srpos=1\&e=\%2D\%2D\%2D\%2D\%2D\%2D-vi20\%2D\%2D1\%2D\%2Dimg-txIN-H\%25e1\%25bb\%2593+S\%25e1\%25bb\%25b9 +Tri\%25e1\%25bb\%2581u

31. Van De N, Le TH, Lien PTH, Eom KS. Current status of taeniasis and cysticercosis in Vietnam. Korean J Parasitol. 2014;52:125-9.

32. Openshaw JJ, Medina A, Felt SA, Li T, Huan Z, Rozelle S, et al. Prevalence and risk factors for Taenia solium cysticercosis in school-aged children: a school based study in western Sichuan, People's Republic of China. PLoS Negl Trop Dis. 2018;12:e0006465.

33. Prasad KN, Verma A, Srivastava S, Gupta RK, Pandey CM, Paliwal VK. An epidemiological study of asymptomatic neurocysticercosis in a pig farming community in northern India. Trans R Soc Trop Med Hyg. 2011;105:531-6.

34. Cherian A, Syam UK, Sreevidya D, Jayaraman T, Oommen A, Rajshekhar V, et al. Low seroprevalence of systemic cysticercosis among patients with epilepsy in Kerala-South India. J Infect Public Health. 2014;7:271-6.

35. Rebecca WP, Eugene II, Joshua K. Seroprevalence of antibodies (lgG) to Taenia solium among pig rearers and associated risk factors in Jos metropolis, Nigeria. J Infect Dev Ctries. 2013;7:67-72. 
36. Mwanjali G, Kihamia C, Kakoko DVC, Lekule F, Ngowi H, Johansen MV, et al. Prevalence and risk factors associated with human Taenia solium infections in Mbozi district, Mbeya region, Tanzania. PLoS Negl Trop Dis. 2013;7:e2102.

37. Garcia-Noval J, Moreno E, de Mata F, Soto de Alfaro H, Fletes C, Craig PS, et al. An epidemiological study of epilepsy and epileptic seizures in two rural Guatemalan communities. Ann Trop Med Parasitol. 2001;95:167-75.

38. Edia-Asuke AU, Inabo HI, Mukaratirwa S, Umoh VJ, Whong CM, Asuke S, et al. Seroprevalence of human cysticercosis and its associated risk factors among humans in areas of Kaduna metropolis, Nigeria. J Infect Dev Ctries. 2015;9:799-805.

39. Dak Lak Provincial People's Committee. An overview about Dak Lak province. 2014; 5/8/2014. https://daklak.gov.vn/web/english/about-daklak.

40. Gabriël S, Dorny P, Mwape KE, Trevisan C, Braae UC, Magnussen P, et al. Control of Taenia solium taeniasis/cysticercosis: the best way forward for Sub-Saharan Africa? Acta Trop. 2017;165:252-60.

41. Winskill P, Harrison WE, French MD, Dixon MA, Abela-Ridder B, Basáñez M-G. Assessing the impact of intervention strategies against Taenia solium cysticercosis using the EPICYST transmission model. Parasit Vectors. 2017:10:73.

42. O'Neal SE, Moyano LM, Ayvar V, Rodriguez S, Gavidia C, Wilkins PP, et al. Ring-screening to control endemic transmission of Taenia solium. PLoS Negl Trop Dis. 2014;8:e3125.

Ready to submit your research? Choose BMC and benefit from:

- fast, convenient online submission

- thorough peer review by experienced researchers in your field

- rapid publication on acceptance

- support for research data, including large and complex data types

- gold Open Access which fosters wider collaboration and increased citations

- maximum visibility for your research: over $100 \mathrm{M}$ website views per year

At BMC, research is always in progress.

Learn more biomedcentral.com/submissions 